\title{
OCCUPANCY-DRIVEN FACILITY MANAGEMENT AND BUILDING PERFORMANCE ANALYSIS
}

\author{
D. IOANNIDIS ${ }^{1,2}$, S. ZIKOS ${ }^{1}$, S. KRINIDIS ${ }^{1}$, A. TRYFERIDIS ${ }^{1}$, D. TZOVARAS ${ }^{1} \&$ S. LIKOTHANASSIS $^{2}$ \\ ${ }^{1}$ Information Technologies Institute, Centre for Research and Technology Hellas, Greece. \\ ${ }^{2}$ Pattern Recognition Laboratory, Computer Engineering and Informatics, University of Patras, Greece.
}

\begin{abstract}
Accurate and automated real-time building monitoring is a challenging task due to the large number of different parameters that are involved. This paper presents several aspects of a building monitoring and control system that has been developed. The system is based on a multi-sensorial network which is able to capture sustainability-related metrics due to the use of heterogeneous sensors such as occupancy detection sensors, environmental sensors, power consumption sensors and actuators. The availability of occupancy information at room or zone level which is achieved via the occupancy sensors, offers the opportunity to extract valuable information about the way the building is utilized. Furthermore, as the occupancy patterns considerably affect the building's indoor environmental condition and the consumed energy, occupancy estimation turns into a useful tool for the facility manager to analyze and then improve the building's energy performance and enhance the indoor environmental quality by applying control actions through the actuators. The building information is visualized in the spatio-temporal domain to both the facility manager and the occupants via two different components. The system has been evaluated in real-life conditions at a building which is comprised of different types of spaces. Results showed that useful conclusions are drawn when occupancy information is combined with the other monitored parameters.

Keywords: energy conservation, occupancy estimation, sensor network, visualization.
\end{abstract}

\section{INTRODUCTION}

Optimal energy use is a basic principle for sustainable building operation. For this reason, various parameters that can lead to substantial reductions in energy consumption have to be combined effectively, such as energy-efficient building designs, energy saving technologies, informed behavioral choices and optimization based on local climatic conditions [1]. There is high interest for novel solutions that utilize diverse information from smart sensor networks to analyze a building's state. These networks often employ different sensor types for monitoring parameters such as occupancy, environmental variables, the state of appliances etc. Even though there are an increasing number of sensors ready to be used, there is still a lack of low-cost and versatile services for the installation and maintenance of sensors as well as data analysis [2]. Furthermore, the interfaces among heterogeneous sensors must be clear, and interoperability with additional smart sensors has to be assured. Information fragmentation and redundancy that can be caused by the usage of separate and disconnected tools can introduce problems for well-informed decision making [3]. Wireless Sensor Networks are increasingly being used even in domestic environments. For example, Suryadevara et al. [4] present a system that monitors and controls electrical appliances at a home. The management and presentation of the collected data is another challenging issue. The visualization tools have to display detailed information properly categorized, in a format close to human perception. Consequently, the facility managers will be able to effortlessly review and assess the building's state in real-time, and apply the proper control actions if necessary.

Occupancy monitoring in buildings is of high interest. Occupancy information can contribute to the performance analysis of the building, since occupancy affects power 
consumption and indoor environmental conditions. Optimizing these two parameters is an important step towards sustainable building operation. The use of rooms, use of appliances and occupancy hours are some of the user related factors that affect energy consumption in buildings [5]. Rafsanjani et al. [6] present a detailed review of methods and models that are used for analyzing occupants' energy-use behaviors in commercial buildings. Various approaches have been proposed in the literature for occupancy estimation utilizing different sensing hardware. For example, there are studies that utilize occupancy sensors to detect occupancy [7], and studies such as the one presented in ref. [8], which exploit the existing infrastructure by using implicit sensing methods. In relation to the way the data from different sources are combined, rule-based approaches and machine learning based approaches $[9,10]$ have been proposed. In case the occupancy estimations are utilized in a decisionmaking process, it is important to limit the false negative cases and long-lasting false positive intervals to avoid inappropriate actions.

The objective when designing sustainable buildings is to reduce the overall impact of the construction on the natural environment and occupant health. Even though a building may have been designed and constructed according to sustainability principles, proper operation and maintenance are required to remain sustainable. The present paper presents an integrated building monitoring and facility management system which is evaluated at a research institute. The system exposes various aspects of the building's state in the spatiotemporal domain such as the status and power consumption of devices (Heating, ventilation and air conditioning-HVAC systems, lights, etc.), environmental conditions such as temperature and humidity, and real-time occupancy information. Occupancy information, available at building level and as low as room level, allows the facility manager to correlate the various monitored parameters with occupancy. Through the analysis of the data provided, the facility manager can assess whether crucial principles of sustainability are followed to intervene and improve the building performance in case of divergence. The optimization of energy performance and the enhancement of the indoor environmental quality are the two main sustainability principles that can be evaluated when using the system. Energy performance is monitored via the power meters, while the environmental quality is monitored through the carbon dioxide, luminance and other sensors. This study extends the previous work presented in [11]. Occupancy estimation topics have been studied, such as the discovery of efficient sensor combinations and the application of an occupancy model to a different space. Moreover, functionalities that were added for increasing the performance, the usability and the efficiency of the system are presented. Additional aspects of the system that show how occupancy information is engaged in facility management operations are presented and experimental results derived from compound case studies are also provided.

The remainder of the paper is divided into the following sections: Section 2 presents the multi-sensorial network and the system architecture. Section 3 describes the approach for occupancy estimation and other occupancy related topics. The visualization components are presented in Section 4 and evaluation results are demonstrated in Section 5. Lastly, the conclusions and topics for further research are summarized in Section 6.

\section{MULTI-SENSORIAL NETWORK}

The multi-sensorial network includes different sensor types to monitor a wide range of aspects of the building's state. Table 1 summarizes the main characteristics of the different hardware utilized for sensing and control. 
Table 1: Main characteristics of installed sensor types.

\begin{tabular}{llll}
\hline Sensor Type & Usage & Output & Connection Interface \\
\hline PIR motion & Occupancy & Binary state & Via alarm system \\
Magnetic contact & Occupancy & Binary state & Via alarm system \\
$\begin{array}{l}\text { Double-beam/ } \\
\text { Pressure mats }\end{array}$ & Occupancy & Entry / exit & Arduino with Wi-Fi or \\
Acoustic & Occupancy & Sound activity every & Arduino with Wi-Fi or \\
& & 4 seconds & Ethernet \\
RFID antennas & Occupancy & RFID tag detection & Ethernet PC \\
Depth cameras & Occupancy & Number of occupants & Ethernet PC \\
CO 2 & Occupancy / & Concentration in & EnOcean (wireless) \\
Temperature & Environmental & PPM & \\
Humidity & Environmental & ${ }^{\circ}$ C & EnOcean (wireless) \\
Luminance & Environmental & Relative $\%$ & EnOcean (wireless) \\
SmartPlugs & Environmental & Lux & EnOcean (wireless) \\
& Energy cons. / & Power cons., status & ZigBee \\
Water heater sensor/ & Energy cons. / & Power cons., water & Arduino with Ethernet \\
actuator & Control & temperature & \\
Actuators & Control & N/A & EnOcean or OPC server \\
\hline
\end{tabular}

\subsection{Sensor types}

Occupancy is estimated via the use of various sensor types such as passive infrared (PIR) motion detectors, acoustic sensors, door counters, depth cameras etc., where most of them are low-cost sensors. More details about occupancy estimation are presented in Section 3. Furthermore, for each monitored space or zone, several environmental attributes such as carbon dioxide concentration, temperature, humidity and luminance, are logged through the use of the respective sensors. The status and the current power consumption of electrical devices such as printers, coffee makers, monitors and PC towers is retrieved from the Smart Plug each device is attached to. The status of a device is reported on change, while the power consumption is reported every minute. The Smart Plug also allows the control of the connected device. Concerning the monitoring and control of the water heater appliance, a dedicated sensoractuator was developed. It consists of an Arduino board, a temperature sensor, a Current Transformer (CT) sensor, and a relay, and reports the power consumption in Watts along with the water temperature every 5 seconds. In order to be able to control the lights and the HVAC systems, the multi-sensorial network includes on/off switch actuators. Dimmer actuators were installed as well in a multi-occupant office, providing 10 discrete luminous intensity levels.

\subsection{Proper placement of sensors}

Proper placement of the sensors has been taken into consideration during the installation procedure. Apart from detection accuracy, other parameters that were considered are the 
obtrusiveness of the installation, the protection of the equipment and connectivity. For example, Double-beam door counters are installed at a height of about $1.20 \mathrm{~m}$ to minimize the probability of producing double-counts due to arm movement. Moreover, experiments showed that the performance of the Double-beam counter can be improved in case the distance between the two beams can be increased. The maximum allowed distance is the one that allows both beams to be in an interrupted state simultaneously when an occupant passes by the doorway. The experiments were performed at a double door entrance where the distance between the two beam sensors' casings was set to $2.5 \mathrm{~cm}$. Results showed a slight increase in the direction detection rate which was due to the longer time intervals between the break-reset times of the different beams. Another action that was taken to improve the efficiency of the installation is the reuse of equipment. The use of only one Arduino board for controlling two independent Double-beam counters placed at two adjacent entrances has been evaluated to further improve the cost efficiency. The control algorithm has been modified to allow the simultaneous monitoring of the states of the four total beams (two pairs). The produced entry/exit events are stored temporarily in a local memory buffer and when all four beams are in an uninterrupted state, the events are dispatched to the server. In this way, the counter can log simultaneous entries or exits occurring at both entrances simultaneously. However, a minor drawback of the approach is that the dispatch of an event may be delayed when an occupant passes by, while another occupant stands in the other doorway. Information about the placement of the other sensor types can be found in our previous work [11].

In order to decrease the obtrusiveness of the installation, cables were concealed as much as possible via cable management. Moreover, Arduino boards were placed inside plastic case boxes for protection. Keeping a safe distance between a sensor and other electrical equipment is another action that was performed to avoid interference which results in flawed sensorial data. Lastly, each node is connected to the optimal Wi-Fi network based on location and can reconnect if a broken connection is detected.

\subsection{System architecture}

Data that are generated continuously by the low-level heterogeneous sensors are stored into a central database via the use of intermediate hardware and software sub-components. Each one is responsible for the management of specific sensors of the same interface. There are also higher level components, which utilize the data stored in the database to estimate realtime occupancy, visualize the information, and allow controlling devices. These higher level components are presented in the following sections. The overall architecture of the system is depicted in Fig. 1.

\section{OCCUPANCY-RELATED INFORMATION}

In this section, the occupancy estimation and occupancy prediction components are described. Moreover, the methodology that was followed for discovering efficient sensor combinations is presented in addition to the method for occupancy detection at the building level.

\subsection{Occupancy estimation and prediction}

Real-time occupancy estimation in the different spaces of the building is accomplished via the use of the data provided by the installed occupancy sensors. The system supports both group-based (occupancy per space) estimation and individual location extraction (location 


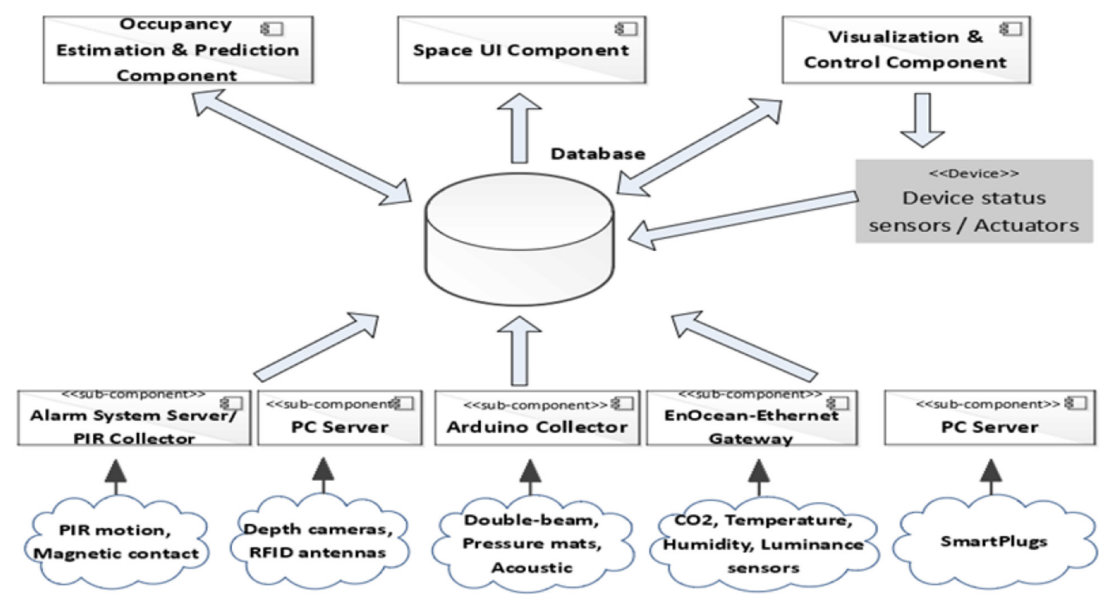

Figure 1: System architecture.

per occupant). The latter maps each occupant to a specific location at any given time, via the use of Radio-frequency identification (RFID) antennas located over entrances, and semipassive tags carried by the occupants. The detailed description of the individual location extraction method is beyond the scope of this paper. Group-based occupancy estimation for a given space can be provided by one of the two following systems: 1) system that utilizes depth camera(s) and 2) system that uses multiple low-cost sensor types. The former system [12] is able to detect human silhouettes in real-time and outputs the estimated number of occupants detected in the monitored area with an average accuracy of about $95 \%$. The latter occupancy estimation system utilizes multiple low-cost sensor types such as door counters, PIR motion detectors etc. to estimate the occupancy of a space. Estimations are produced with the use of a linear chain Conditional Random Field (CRF) probabilistic model [13], which is a discriminative graphical model for labelling sequence data. At each time step $t$, the model takes as input a feature vector (observation) $x_{t}$, where each feature is a numeric value associated with a weight and derived from the raw data of a particular sensor type. The model infers the most probable state $y_{t}$ given the observation (or the most probable sequence of hidden states given an observation sequence). In our domain, each occupancy class, which is represented by an integer value, is modeled as a state and can be either presence/absence detection or occupancy density (5 levels) or the number of occupants (0...maxCapacity), based on the model definition. The probability $\mathrm{p}(\mathrm{y} \mid \mathrm{x})$ of producing a state sequence $y$ given an observation sequence $x$ can be computed by eqn (1):

$$
p(y \mid x)=\frac{1}{Z(x)} \exp \left\{\sum_{k=1}^{K} \lambda_{k} f_{k}\left(y_{t}, y_{t-1}, x_{t}\right)\right\}
$$

where $f_{k}$ is a feature function, $K$ is the number of features and $\lambda_{k}$ is a learned weight associated with feature $f_{k}$. Z(x) is the normalization function (eqn (2)).

$$
Z(x)=\sum_{y} \exp \left\{\sum_{k=1}^{K} \lambda_{k} f_{k}\left(y_{t}, y_{t-1}, x_{t}\right)\right\}
$$

According to the online operation, which is selected in this case, the features that are based on sensorial data are computed and passed to the CRF classifier at each time step. The 
classifier component updates the probabilities for each occupancy class and returns the estimated state, which is the class with the highest probability. A training phase is required in advance to compute the weights and create a model. A sequence of observations along with the actual occupancy label (ground truth) per each observation are provided as input for learning the model parameters by applying maximum-likelihood estimation. More details on the occupancy estimation and a feature extraction method can be found in the recent work [14].

In addition to occupancy estimation, information about expected occupancy in the future is also extracted by the occupancy prediction sub-component. The developed framework, which is described in Adamopoulou et al. [15], supports short-term and mid-term prediction. Current occupancy information is utilized along with models created using historical occupancy data per space to produce the prediction. The facility manager can exploit the provided occupancy prediction information to apply actions for improving the comfort of occupants (switch on HVAC for pre-heating/cooling) or for decreasing the energy consumption (switch off an HVAC).

\subsection{Efficient sensor setups}

An interesting research topic is the discovery of optimal sensor combinations for estimating the occupancy of the selected granularity (presence detection, occupancy density, number of occupants) in a particular space. Besides performance, other aspects can be also taken into account when selecting a sensor combination, such as the cost, the privacy efficiency and the obtrusiveness of installation. In a resource-constrained scenario, where high cost efficiency is the top priority, the use of the most inexpensive combination that meets the performance requirements is preferred. On the contrary, in a performance-driven scenario the cost efficiency can be ignored and the most accurate sensor combination is selected. Generally, accuracy increases with increasing number of different sensor types, however, total cost increases too. The methodology that was followed for discovering efficient sensor combinations for each type of space is the following: a) Initially, the different sensor types were installed. b) Several CRF models were created (all trained on the same dataset) where each model utilized a specific combination of sensors. c) The estimations of all models were evaluated on the same testing dataset. d) Based on the performance and the cost criteria, the most efficient combination was defined. The results regarding the efficient sensor setups are presented in Section 5.

In some cases, the acquisition of ground truth for training a model for a particular space may not be feasible. Thus, it is worthwhile to evaluate the performance of an already trained model when applied to another similar space using the same sensor types. A framework for cross-space occupancy modeling through ambient sensing and relationship learning is presented in [16]. In our case, the presence detection model created for the meeting room, utilizing a PIR motion and an Acoustic sensor, was applied to the conference room. The latter space is larger and can accommodate up to 40 occupants but is quite similar to the meeting room in terms of usage. The detection accuracy of the model was very high, as shown in Table 2.

In case the maximum performance is required, an occupancy fusion method has been developed which combines the output of the depth cameras system with the output of the multi-sensorial system. The multi-sensorial system can operate in a complementary mode for a selected space. In this mode, the system compares its estimation with the occupancy estimated from the depth cameras in real-time to detect possible divergence. The drawback of 
Table 2: Presence detection (model trained for meeting room).

\begin{tabular}{lll}
\hline Location & Sensors & Accuracy \\
\hline Meeting room & PIR, Acoustic & $98.0 \%$ \\
Conference room & PIR, Acoustic & $97.5 \%$ \\
\hline
\end{tabular}

this particular method is the increased cost which is introduced due to the use of additional equipment, since the space of interest must be monitored by both systems concurrently. The method has been tested in the building's kitchen to reduce false positive intervals as in certain cases it has been observed that the depth camera system may still estimate the monitored space as occupied for a considerable time after the departure of all occupants. A CRF model for presence detection using an acoustic and a PIR motion was utilized. The CRF-based multi-sensorial system intervenes by setting occupancy to zero only in the case when the depth camera system detects occupant(s) for more than 10 minutes and the multi-sensorial system estimates the space as empty throughout this time period. The results derived from the real-time operation of the method showed that the corrections in occupancy were justified. A faster transition to the unoccupied state through the application of the occupancy fusion method can contribute to higher energy savings due to more efficient management of devices.

\subsection{Occupancy detection at building level}

Knowledge of the occupancy status of a building is important since the use of publicly accessed appliances such as printers and water heaters is correlated with the building's occupancy. In order to detect whether the monitored building is occupied or not, an event-based method which does not require training has been developed and implemented. The method utilizes the real-time events received from all the alarm system's PIR motion and magnetic contact sensors that are installed. The sensors were initially separated into logical groups, depending on their location. One group includes the sensors installed in closed spaces (e.g. offices) and the other group includes the sensors located at open-spaces (corridors). The sensor ID and the timestamp of the last event are retrieved and stored. The building is estimated as unoccupied in case no event has been received for the last $\mathrm{N}$ minutes, where $\mathrm{N}=90 \mathrm{~min}$ utes and $\mathrm{N}=30$ minutes for the sensors installed in closed spaces and the sensors installed in corridors, respectively. Furthermore, the alarm system's arm and disarm events are also monitored. When the alarm system is armed, the building occupancy is set to 0 . On the contrary, when the alarm system is disarmed, a 5-minute interval is considered (unoccupied state) to allow the security staff to leave the building, which will be estimated as occupied as soon as the first motion event is received. The detection performance that was achieved was very satisfactory, as the method reduces the false positive cases considerably and avoids false negatives during the working hours.

\section{VISUALIZATION AND FACILITY MANAGEMENT}

The Space UI component presents the current state of a particular space. The status of lights and HVAC, total power consumption, indoor environmental conditions (e.g. temperature) and current occupancy are shown to the occupants in a user-friendly format. An example is depicted in Fig. 2. The presentation of the data must be clear, configurable, and interactive to allow the facility manager to perceive possible compound relations among the different 


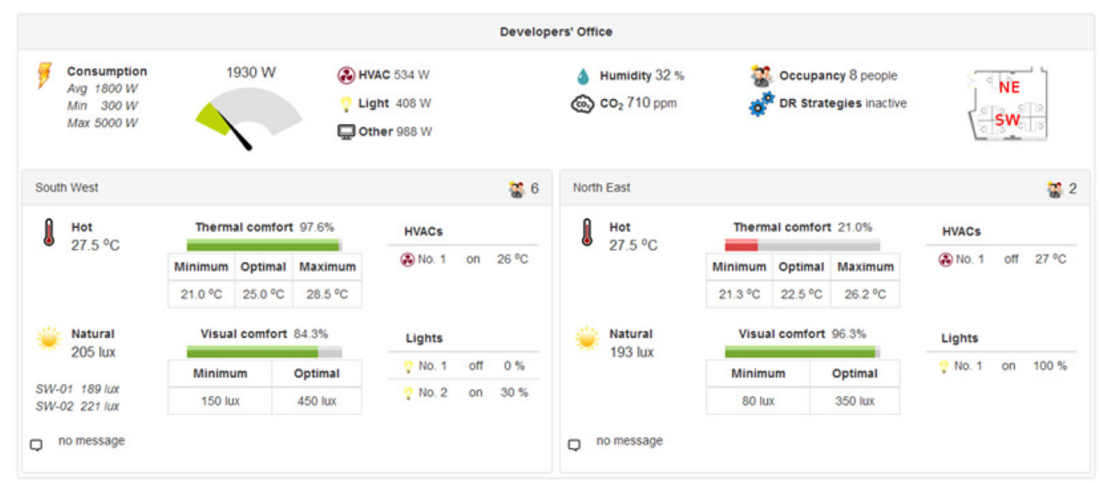

Figure 2: SpaceUI in a multi-occupant office.

measurements. Consequently, the facility manager's visualization and control component adopt principles from the field of Visual Analytics [17]. The highly configurable advanced visual interfaces allow the facility manager to evaluate the state and perform the required actions. The component visualizes both real-time and historical information. In the first case, a general view of the condition (occupancy, total consumption) of all spaces is provided. The facility manager can select a particular space to view more detailed information, such as the status of devices and the environmental condition, and control devices via the actuators. Through the presentation of historical data, the facility manager can discover patterns and performance issues that need to be addressed, by selecting the desirable parameters, time interval, and time step resolution. The visualization components are able to present the percentage of thermal and visual comfort metrics for the selected monitored area. Thermal comfort is computed based on registered user preference and the current value of the corresponding indoor temperature sensor. The visual comfort, likewise, is computed based on the indoor luminance level reported by the luminance sensor.

Due to the large number of the installed sensors, a monitoring component has been developed to inform the facility manager via e-mail about abnormal delays in the reception of events. In case the time interval since the last received event exceeds a threshold, the sensor ID is added to the list and highlighted, as it is shown in Fig. 3. The sensors are categorized based on type, and a different time interval threshold is assigned to each category.

Regarding the operation and control of the monitored devices, a component has been developed that allows the automatic control of the water heater. The appliance is controlled based on the current water temperature and the desired set water temperature. The latter is dynamic and is determined automatically between a minimum and a maximum value, based on electricity pricing during the day and building occupancy information. More particularly,

\begin{tabular}{|c|c|c|c|c|c|c|}
\hline \multicolumn{2}{|c|}{ Start } & Stop & \multicolumn{2}{|r|}{$\Gamma$ Alarm via e-mail } & Check every (minutes) & $15 \div$ \\
\hline & & Sensor ID & Last Event Time & Value & Location & $\Delta$ \\
\hline 1 & Dim_ & immerStatus & $\begin{array}{l}2015-12-07 \\
08: 26: 16.485\end{array}$ & on & Developer_SW_01 & \\
\hline 2 & Dim_ & immerStatus & $\begin{array}{l}2015-12-07 \\
08: 27: 54.293\end{array}$ & on & Developer_SW_01 & \\
\hline
\end{tabular}

Figure 3: System status monitoring and alert. 
a set temperature is determined per 15 minutes at the start of the day for the entire day. Lower electricity price results in a higher selected set temperature in order for the appliance to heat the water when energy is cheaper, exploiting the appliance's thermal inertia. Prediction of the building's occupancy for the next 12 hours is utilized to define the time interval during which the use of hot water is expected. After the preferred temperatures have been set, the current temperature is monitored to send a) a switch on command in case it drops below the preferred temperature or b) a switch off command in the opposite case.

\section{EXPERIMENTAL RESULTS FOR BUILDING PERFORMANCE ANALYSIS}

The multi-sensorial network is deployed to more than 10 spaces, such as offices of different capacities, corridors, the building's kitchen, a meeting room etc. In some cases, each space is divided into two or more zones. The installation includes more than 50 sensors for occupancy estimation, about 30 environmental sensors, 15 controllable HVAC units and about 60 controllable devices and appliances. The overall experiment has been carried out over a year. Figure 4 shows the locations of the different sensors installed in three of the monitored spaces, Researchers' office, Administration office, and Kitchen.

As far as occupancy estimation is concerned, evaluation results revealed sensor setups which yielded high efficiency in terms of both performance and cost, shown in Table 3. Accuracy is the main performance metric when detecting presence, while the normalized root-mean-square error (NRMSE) is used when estimating the number of occupants or occupancy density. It is noted that in two cases the use of a single sensor type alone yielded high performance when detecting the presence.
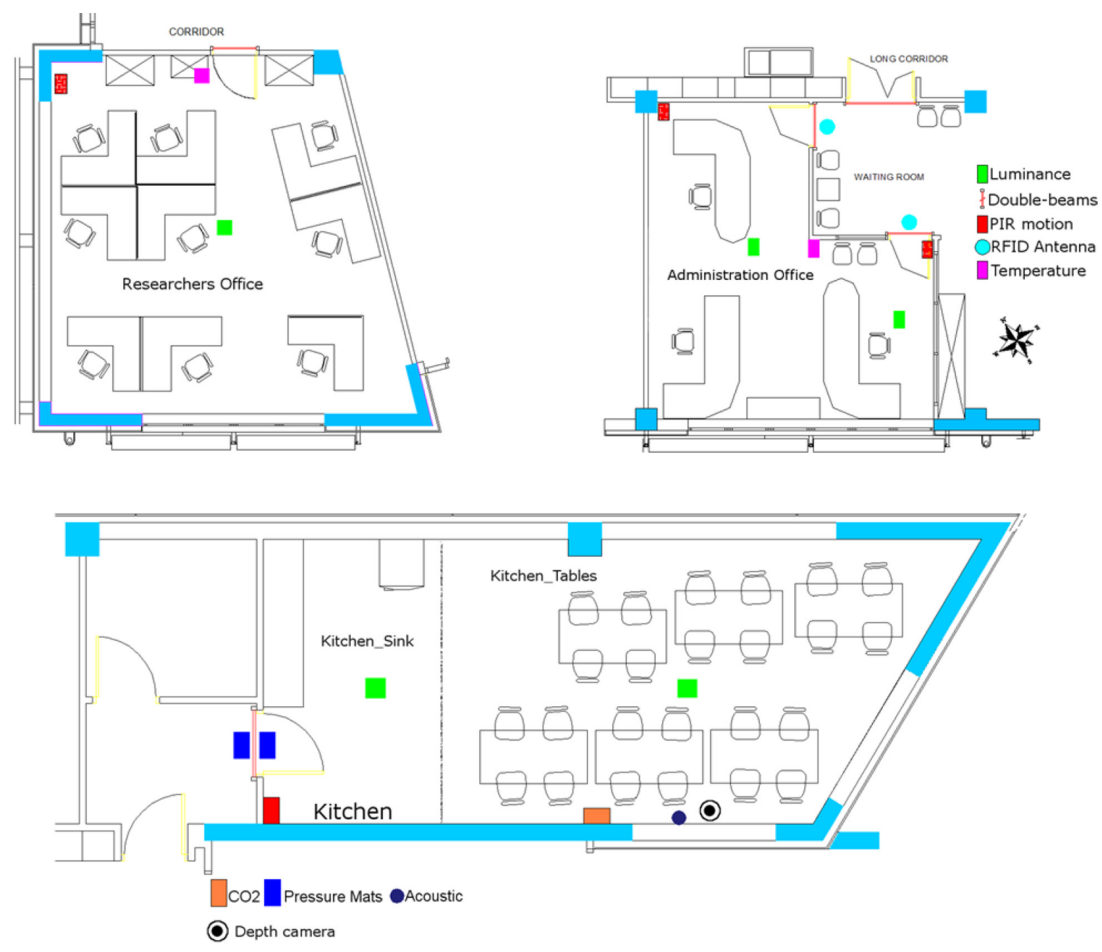

Figure 4: Locations of sensors in two multi-occupant offices and in kitchen. 
Table 3: High performance cost-efficient sensor setups.

\begin{tabular}{|c|c|c|c|}
\hline Location & Estimation type & Sensors & Accuracy/NRMSE \\
\hline $\begin{array}{l}\text { Open-space rest } \\
\text { area }\end{array}$ & Presence detection & Acoustic & $84 \%$ (Accuracy) \\
\hline \multirow[t]{2}{*}{ Meeting room } & $\begin{array}{l}\text { Exact number or } \\
\text { Density }\end{array}$ & Double-beam, Acoustic & 0.105 (NRMSE) \\
\hline & Presence detection & PIR motion, Acoustic & 98\% (Accuracy) \\
\hline \multirow[t]{2}{*}{ Kitchen } & Density & $\begin{array}{l}\text { Double-beam/Pressure mats, } \\
\text { Acoustic }\end{array}$ & 0.12 (NRMSE) \\
\hline & Presence detection & PIR motion, Acoustic & 94\% (Accuracy) \\
\hline \multirow{2}{*}{$\begin{array}{l}\text { Multi-occupant } \\
\text { office }\end{array}$} & Density & Double-beam/Pressure mats & 0.121 (NRMSE) \\
\hline & Presence detection & PIR motion & 95\% (Accuracy) \\
\hline $\begin{array}{l}\text { Single-occupant } \\
\text { office }\end{array}$ & Exact number & Double-beam, PIR motion & 0.15 (NRMSE) \\
\hline
\end{tabular}

For the evaluation of the received sensor readings and the system integration, historical data that are provided by different sources were requested, compared and checked for consistency. Figure 5 shows the variation of the estimated number of occupants as well as the HVAC status and external temperature over three consecutive days in the meeting room. The power consumption of the lights during the same time period is depicted in Fig. 6, where each curve corresponds to the lights controlled by the respective switch. From the two figures it can be observed that in several meetings that took place, the occupants did not turn the HVAC on. The number of occupants varied among different meetings and occupants turned on both light switches most of the times. The daily short occupied periods in the morning are caused by the cleaning staff.

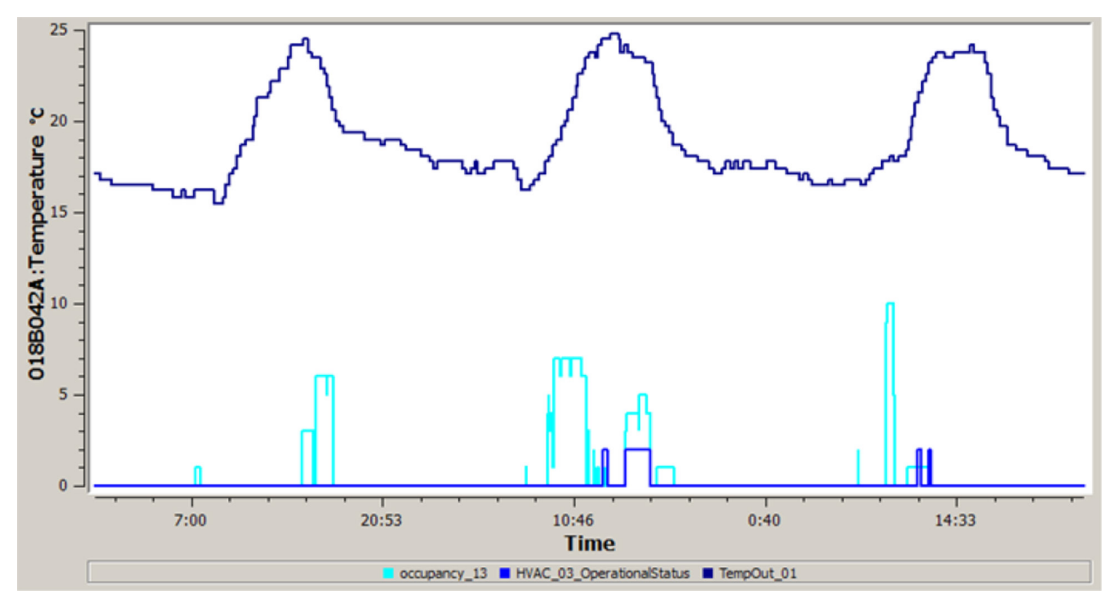

Figure 5: Occupancy in meeting room versus HVAC status and external temperature for 3 days. 


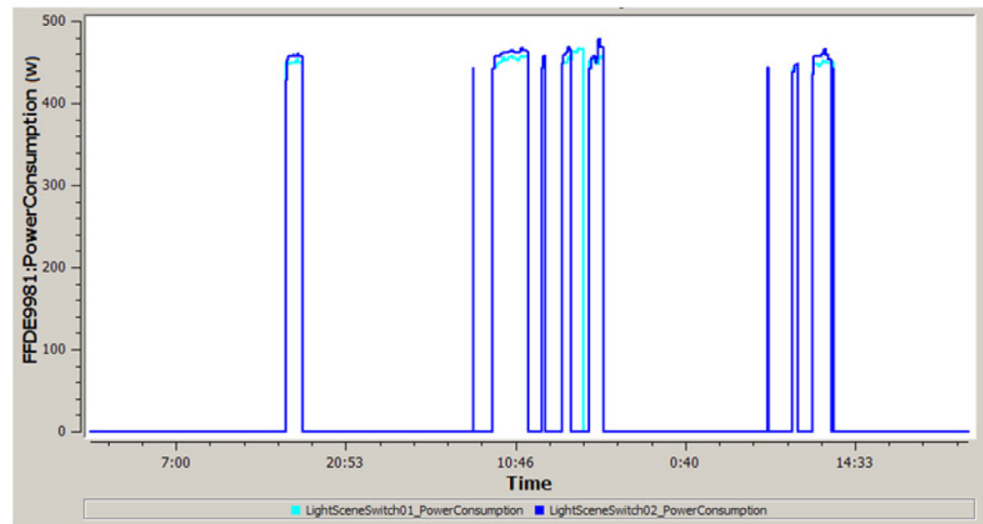

Figure 6: Power consumption (W) of lights in meeting room for the same 3 consecutive days.

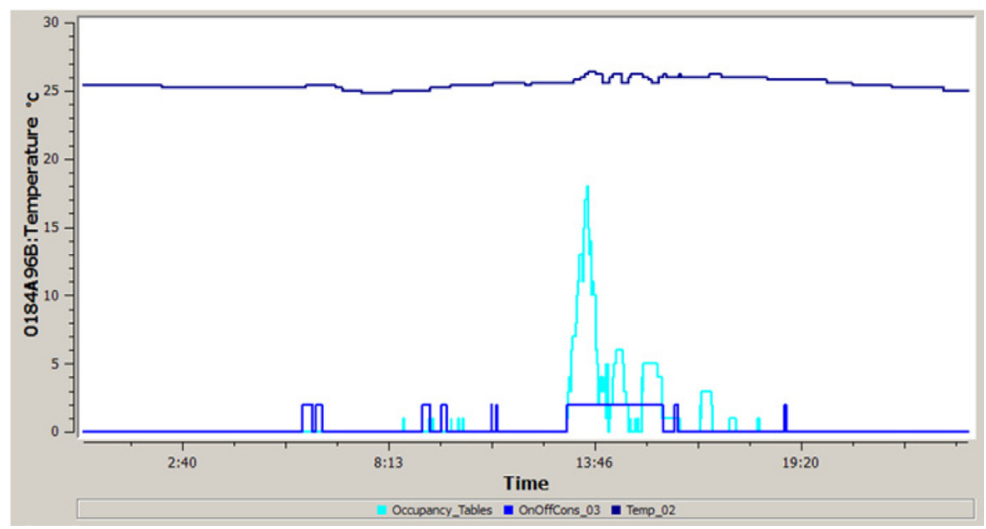

Figure 7: Occupancy versus light status and ambient temperature during a day in Kitchen_Tables.

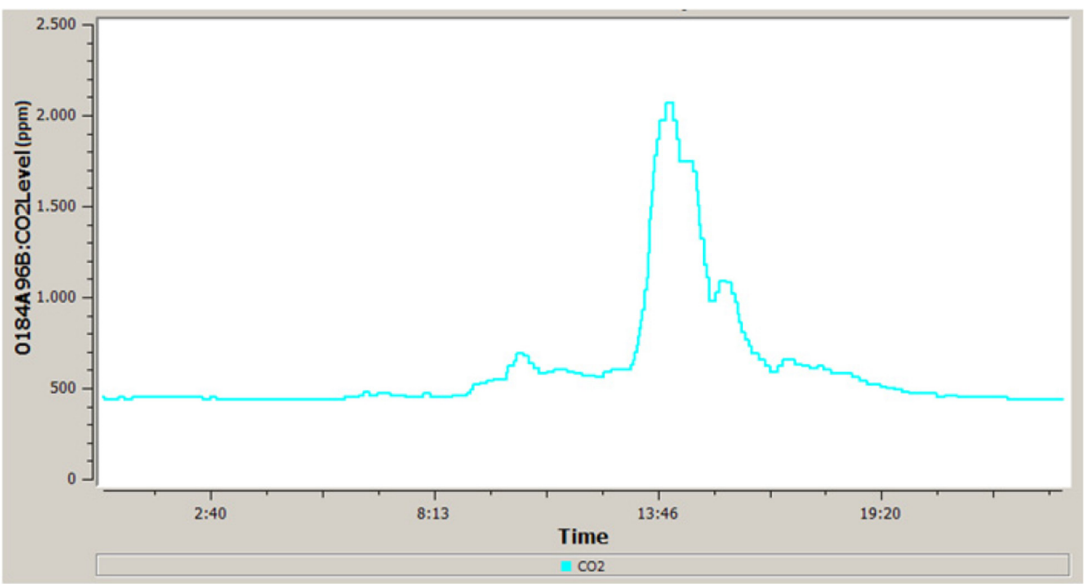

Figure 8: $\mathrm{CO}_{2}$ concentration (ppm) during a single day in Kitchen. 
Figures 7 and 8 show historical data concerning the Kitchen_Tables zone which is part of the building's kitchen. Figure 7 presents the estimated number of occupants, light status and ambient temperature throughout a day. The carbon dioxide concentration for the same time period is presented in Fig. 8. We observe that there were occupied periods during which the light was switched off, indicating that the occupants were satisfied with the luminance provided by the lights of the adjacent zone. High $\mathrm{CO}_{2}$ levels, about $2000 \mathrm{ppm}$, were observed at lunch time, that indicates that the ventilation system was turned off resulting in poor air quality.

\section{CONCLUSIONS AND FURTHER RESEARCH}

In this paper, an integrated occupancy driven system for building facility management has been presented. The system fuses information from many different sources through the use of a multi-sensorial network and allows the control of monitored devices. Presentation of information is performed both at the user level and at the facility manager level. Evaluation results showed that the system contributed to the analysis of monitored building's performance via the presentation of historical information, and helped the facility manager to identify possible undesirable indoor environmental conditions and periods of wasted energy consumption, which is an important step towards sustainable building operation. As a future work, we plan to test facility management functionalities for fully automated control of appliances. Moreover, we aim to utilize color coding to the facility manager's visualization component for highlighting the temporal aspect of occupancy.

\section{REFERENCES}

[1] Kumar, P. \& Morawska, L., Energy-pollution nexus for urban buildings. Environmental Science and Technology, 47(14), pp. 7591-7592, 2013. https://doi.org/10.1021/es402549p

[2] Kumar, P., Martani, C., Morawska, L., Norford, L., Choudhary, R., Bell, M. \& Leach, M., Indoor air quality and energy management through real-time sensing in commercial buildings. Energy and Buildings, 111, pp. 145-153, 2016. https://doi.org/10.1016/j.enbuild.2015.11.037

[3] Gursel, I., Sariyildiz, S., Akin, Ö. \& Stouffs, R., Modeling and visualization of lifecycle building performance assessment. Advanced Engineering Informatics, 23(4), pp. 369417, 2009. https://doi.org/10.1016/j.aei.2009.06.010

[4] Suryadevara, N.K., Mukhopadhyay, S.C., Kelly, S.D.T. \& Gill, S.P.S., WSN-based smart sensors and actuator for power management in intelligent buildings. Mechatronics, IEEE/ASME Transactions on, 20(2), pp. 564-571, 2015.

[5] Olivia, G.S. \& Christopher, T.A., In-use monitoring of buildings: An overview and classification of evaluation methods. Energy and Buildings, 86, pp. 176-189, 2015. https://doi.org/10.1016/j.enbuild.2014.10.005

[6] Rafsanjani, H.N., Ahn, C.R. \& Alahmad, M., A review of approaches for sensing, understanding, and improving occupancy-related energy-use behaviors in commercial buildings. Energies, 8(10), pp. 10996-11029, 2015. https://doi.org/10.3390/en81010996

[7] Kuutti, J., Saarikko, P. \& Sepponen, R.E., Real time building zone occupancy detection and activity visualization utilizing a visitor counting sensor network. Proceeding of 
the 11th International Conference on Remote Engineering and Virtual Instrumentation (REV), IEEE, pp. 219-224, 2014.

https://doi.org/10.1109/rev.2014.6784260

[8] Corna, A., Fontana, L., Nacci, A.A. \& Sciuto, D., Occupancy detection via iBeacon on Android devices for smart building management. Proceeding of the 2015 Design, Automation \& Test in Europe Conference \& Exhibition, EDA Consortium, pp. 629-632, 2015. https://doi.org/10.7873/date.2015.0753

[9] Ekwevugbe, T., Brown, N., Pakka, V. \& Fan, D., Real-time building occupancy sensing using neural-network based sensor network. Proceeding of the 7th International Conference on Digital Ecosystems and Technologies, IEEE, pp. 114-119, 2013. https://doi.org/10.1109/dest.2013.6611339

[10] Lu, J., Sookoor, T., Srinivasan, V., Gao, G., Holben, B., Stankovic, J., Field, E. \& Whitehouse, K., The smart thermostat: using occupancy sensors to save energy in homes. Proceeding of the 8th ACM Conference on Embedded Networked Sensor Systems, pp. 211-224, 2010.

https://doi.org/10.1145/1869983.1870005

[11] Zikos, S., Tryferidis, A., Krinidis, S., Ioannidis, D., Tzovaras, D. \& Likothanassis, S., Multi-sensorial network placement for building performance analysis \& evaluation and facility management. presented in International Conference on Building Information Modelling (BIM) in Design, Construction and Operations, Bristol, UK, 09-11 September 2015.

[12] Ioannidis, D., Krinidis, S., Stavropoulos, G., Tzovaras, D. \& Likothanassis, S., Fullautomated acquisition system for occupancy and energy measurement data extraction. Proceeding of the Symposium on Simulation for Architecture and Urban Design, Society for Computer Simulation International, p. 15, April 13-16, 2014.

[13] Lafferty, J., McCallum, A. \& Pereira, F.C., Conditional random fields: Probabilistic models for segmenting and labelling sequence data. Proceeding of 18th International Conference on Machine Learning, Morgan Kaufmann, San Francisco, CA, pp. 282289, June 2001.

[14] Zikos, S., Tsolakis, A., Meskos, D., Tryferidis, A. \& Tzovaras, D., Conditional Random Fields-based approach for real-time building occupancy estimation with multi-sensory networks. Automation in Construction, 68, pp.128-145, 2016. https://doi.org/10.1016/j.autcon.2016.05.005

[15] Adamopoulou, A.A., Tryferidis, A.M. \& Tzovaras, D.K., A context-aware method for building occupancy prediction. Energy and Buildings, 110, pp. 229-244, 2016. https://doi.org/10.1016/j.enbuild.2015.10.003

[16] Yang, Z. \& Becerik-Gerber, B., Cross-space building occupancy modeling by contextual information based learning. Proceeding of the 2nd ACM International Conference on Embedded Systems for Energy-Efficient Built Environments, pp. 177-186, 2015. https://doi.org/10.1145/2821650.2821668

[17] Thomas, J.J. \& Cook, K., A visual analytics agenda. Computer Graphics and Applications, 26(1), pp. 10-13, 2006. https://doi.org/10.1109/MCG.2006.5 